\title{
Anterograde Intra-Arterial Urokinase Injection for Salvaging Fibular Free Flap
}

\author{
Dae-Sung Lee, Sun-Il Jung, Deok-Woo Kim, Eun-Sang Dhong \\ Department of Plastic and Reconstructive Surgery, Korea University Guro Hospital, Korea University College of Medicine, Seoul, Korea
}

\begin{abstract}
We present a case of a 57-year-old male patient who presented with squamous cell carcinoma on his mouth floor with cervical and mandibular metastases. Wide glossectomy with intergonial mandibular ostectomy, and sequential reconstruction using fibular osteomyocutaneous free flap were planned. When the anastomosis between the peroneal artery of the fibular free flap and the right lingual artery was performed, no venous flow was observed at the vena comitans. Then re-anastomosis followed by topical application of papaverine and lidocaine was attempted. However, the blood supply was not recovered. Warm saline irrigation over 30 minutes was also useless. Microvascular thromboses of donor vessels were clinically suspected, so a solution of 100,000 units of urokinase was infused once through a 26-gauge angiocatheter inserted into the recipient artery just at the arterial anastomotic site, until the solution gushed out through the flap vena comitans. Immediately after the application of urokinase, arterial flow and venous return were restored. There were no complications during the follow-up period of 11 months. We believe that vibrating injuries from the reciprocating saw during osteotomies and flap insetting might be the cause of microvascular thromboses. The use of urokinase may provide a viable option for the treatment of suspicious intraoperative arterial thrombosis.
\end{abstract}

Keywords Salvage therapy / Free tissue flaps / Tissue plasminogen activator
Correspondence: Eun-Sang Dhong

Department of Plastic and

Reconstructive Surgery, Korea

University Guro Hospital, Korea University College of Medicine,

148 Gurodong-ro, Guro-gu, Seoul 152-703, Korea

Tel: $+82-2-2626-1197$

Fax: +82-2-852-7484

E-mail: prsdhong@korea.ac.kr

No potential conflict of interest relevant to this article was reported.

\section{INTRODUCTION}

Microvascular free flap transfer has become a standard procedure in the management of complex bony and soft tissue defects, with overall success rates of $95 \%$ to $99 \%[1,2]$. Flap failure is often due to microvascular or peri-anastomotic thrombosis that can cause substantial and irreversible damage to the microcirculation.Therefore, the high success rates for free flap transfer have contributed to the development of techniques for meticulous dissection of flap elevation, anastomosis, and postoperative monitoring and management $[1,2]$. Some factors have been named as progenitors in the development of microvascular thrombosis. Mechanical stress at the anastomotic junction and vasospasm caused by hypothermia and hypotension cause the incidence to increase [3]. Although microvascular thrombosis takes place in a small number of patients, early monitoring and prompt intervention can usually salvage a compromised flap, thereby preventing flap loss $[1,2]$.

Since pharmacological thrombolysis was first used in the $1960 \mathrm{~s}$ for intravenous treatment of pulmonary embolism, thrombolytic therapy has become the treatment of choice for thrombus in coronary, pulmonary, peripheral, and visceral arteries. Pharma- 
cological thrombolysis was first used in microvascular free-tissue transfer in 1987, when Schubert et al. [4] and Lipton and Jupiter [5] reported on the salvage of two free flaps with streptokinase. Since then, the use of thrombolytic agents such as recombinant tissue plasminogen activator (rt-PA), urokinase, streptokinase, and anisoylated plasminogen-streptokinase-activator complex has been shown to be an effective adjunctive measure for salvage of microvascular thrombosis in animal studies [6,7]. However, few case reports have substantiated their use in humans. We present the case of urokinase administration for clinically suspicious emergency intraoperative arterial thrombosis. We were able to applying thrombolytic therapy as reported in previous papers to salvage our case.

\section{CASE}

A 57-year-old male patient came to our attention for squamous cell carcinoma on his mouth floor with cervical and mandibular metastases. Wide glossectomy with intergonial mandibular ostectomy, and sequential reconstruction using fibular osteomyo- cutaneous free flap were planned. Initially, extended radical neck dissection was performed by head and neck surgeons. When the patient's tongue and mandible were completely exposed, severe dysmorphism and adhesion were observed in the surrounding soft tissue (Fig. 1).

The left fibular osteomyocutaneous free flap was elevated, which included the part of the fibular bone for mandibular reconstruction and a skin paddle measuring $8 \mathrm{~cm} \times 18 \mathrm{~cm}$ for reconstructing the floor of the mouth (Fig. 2A). Three osteoseptocutaneous perforators, which were identified by Doppler preoperatively, supplying the skin paddle and the lateral part of the soleus muscle, were included in the elevated flap. Before the fibular free flap was transferred, an ostectomy of the fibular bone to the neo-mandible was carried out using a reciprocating saw and reconstruction plate (Fig. 2B). The peroneal artery, with a diameter of $1.5 \mathrm{~mm}$, was anastomosed in an end-to-end fashion to the well-matched right lingual artery. The warm ischemic time from flap division to initial arteriorrhaphy was 1 hour. After the arteriorrhaphy, the flap was pale, and neither color improvement nor venous return was observed after the completion of

Fig. 1. Squamous cell carcinoma on the lower face

(A) Intraoperative photograph demonstrating dysmorphism of the mandible and surrounding soft tissue. (B) Gross specimen of total tongue mass excised with intergonial mandibular bone.
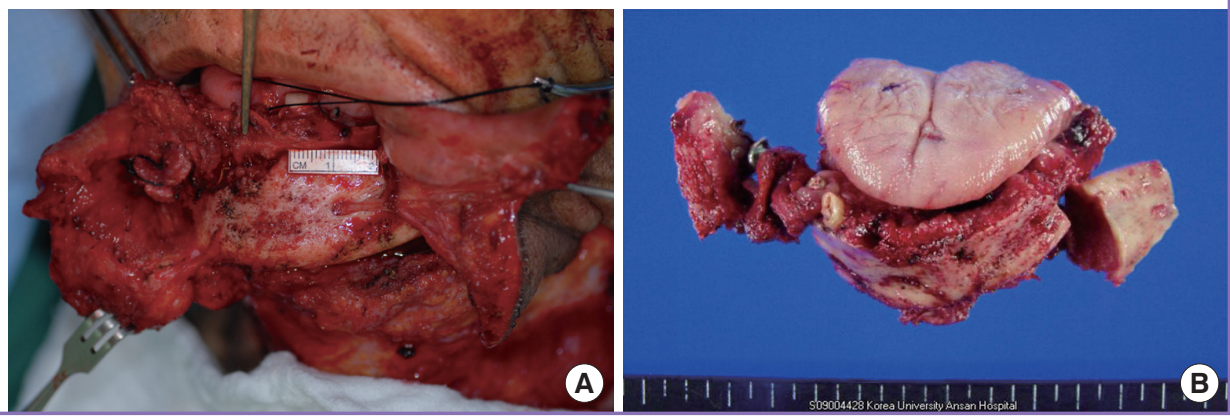

\section{Fig. 2. Flap elevation and fabrication}

Fibular osteomyocutaneous free flap and mandibular reconstruction were performed. (A) The completed flap elevation. Note the part of the fibular bone for mandibular reconstruction and the skin paddle measuring $8 \mathrm{~cm} \times 18 \mathrm{~cm}$ for reconstruction of the floor of the mouth. (B) Appearances after osteotomy of the fibular bone.
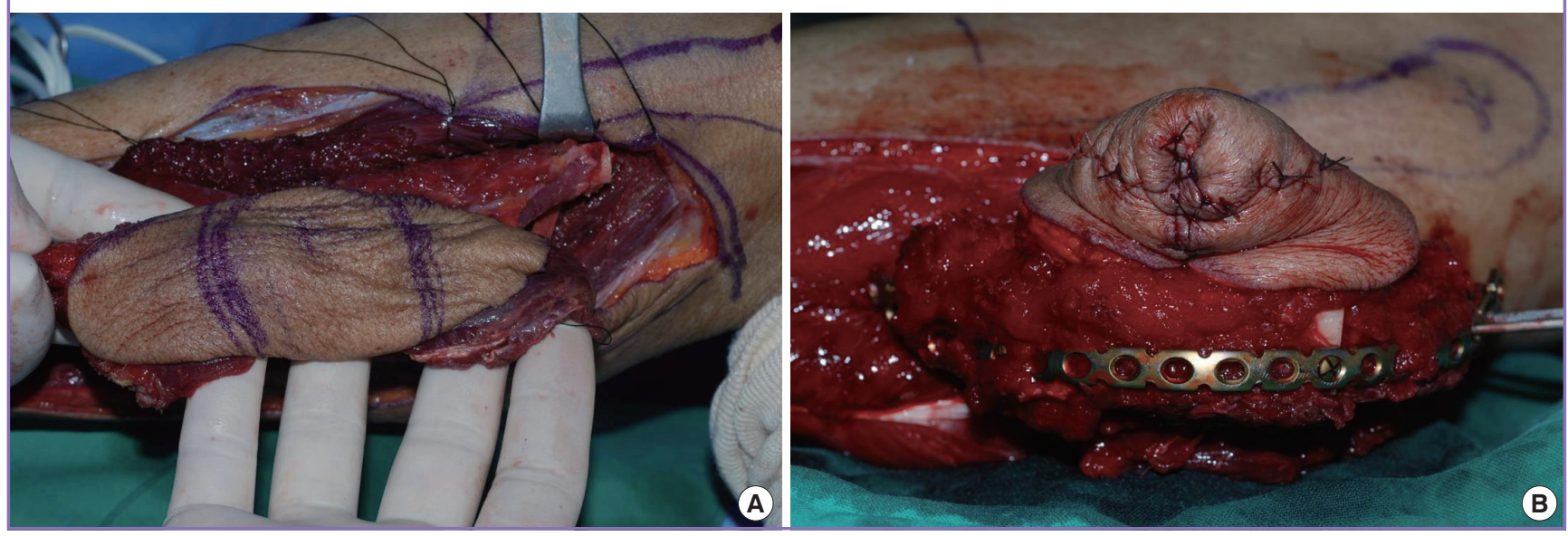
the anastomosis. A localized exploration of the anastomoses was initially performed, showing that the anastomotic junction was patent. Although a topical application of papaverine and lidocaine was done, the blood supply was not recovered. Warm saline irrigation for more than 30 minutes was also useless. To seek a solution, an arterial re-anastomosis was performed, followed by repeated applications of these anti-spasmodic agents, which still gave a negative result. Assessment of vessel patency by Doppler and a milking test was performed, and the arterial anastomotic junction was carefully observed. Thrombectomy and systemic heparin administration were not performed because simple clot or small amounts of clot were absolutely not

\section{Fig. 3. Microvascular free flap salvage process}

Our microvascular free flap salvage process for vascular thrombosis.

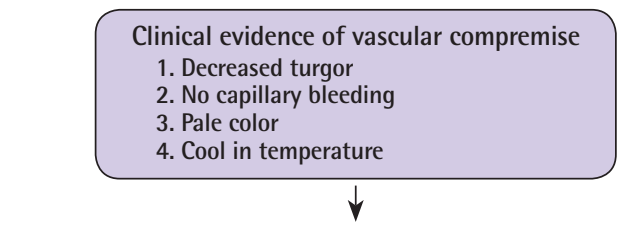

Re-exploration \& careful inspection of anastomotic vessels

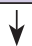

Topical application of papaverine and lidocaine over 30 minutes

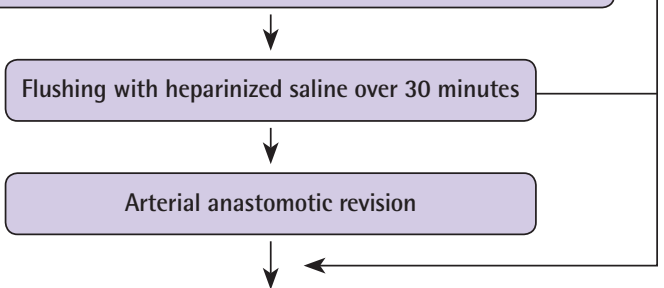

Confirmation of absence of technical errors and vascular spasms, suspicious arterial microthrombus

\section{$\downarrow$}

100,000 IU Urokinase injection via arterial anastomotic juction, reestablish the flow

Fig. 4. Intraoperative view after flap revascularization

Appearance after flap transfer and neomandible recreation. Microvascular anastomoses were performed with the left peroneal artery/concomitant vein and right lingual artery/ right thyroidal vein. Immediately after the application of urokinase, arterial flow was restored and improvement of the flap's color was observed. encountered by the milking test. Although the pedicle had suffcient arterial filling, the flap was still pale with no recovery of venous return within the surgical field. We confirmed there was no technical error. As the arterial anastomosis was definitely patent, microvascular thromboses of the donor vessels under nonvisible area were clinically suspected (Fig. 3). As the ischemic time was becoming prolonged, a subjective test such as intraoperative angiography could not be carried out. After ruling out many possible causes of flap ischemia, the decision was made to use a thrombolytic agent as the last resort.

Two stitches at the arterial anastomotic site were released, and a bolus of 100,000 units of urokinase was infused once anterogradely through a 26-gauge angiocatheter. Within one minute after the application of urokinase, the arterial flow and venous return were restored. Improvement of the flap's color was noted and the urokinase solution gushed out through the flap vena comitans (Fig. 4). Arterial blood reperfusion was performed for over 2 hours. Finally, venorrhaphy was performed with a peroneal vein and right thyroidal vein. There were no further problems nor systemic effects of the thrombolysis and no other treatment was required. The flap survived with a follow-up of 11 months (Fig. 5). We have concluded that vibrating injuries of the reciprocating saw during flap fabrication and insetting might have been the cause of the microvascular thromboses.

\section{DISCUSSION}

Microvascular free tissue transfer is a reliable, established technique in reconstructive surgery. Even at centers with very high free flap success rates, vascular complications of arterial and/ or venous pedicle thrombosis can occur in up to $10 \%$ to $15 \%$ of cases $[1,2]$. Pedicle thrombosis remains a challenge for the microvascular surgeon. Arterial thrombosis typically occurs early in the intraoperative and postoperative course, while venous thrombosis can present with more temporal variability because of the slower flow rate. Management to prevent this pedicle

\section{Fig. 5. Postoperative views}

(A) Endoscopic view of the mouth 11 months after the surgery. (B) Clinical photo 11 months after the surgery.

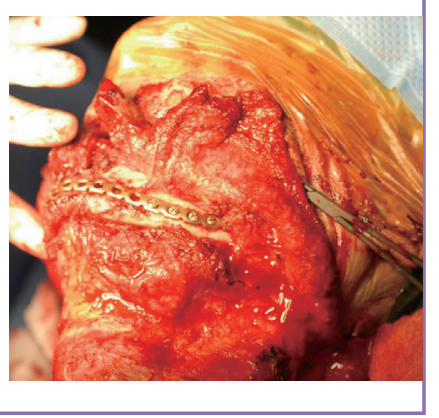

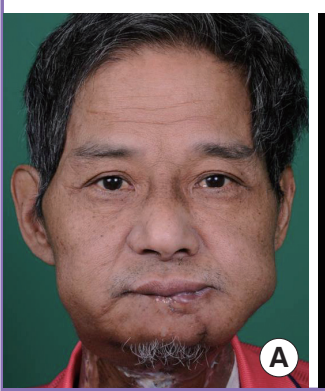

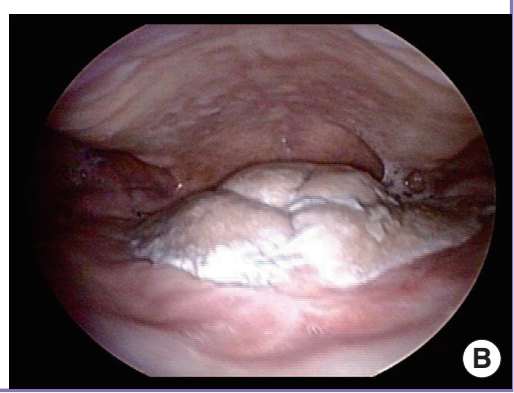


thrombosis include careful patient selection, careful recipient vessel selection, pharmacological prophylaxis, meticulous surgical technique in flap harvesting, vessel preparation and microsurgical anastomosis, and careful postoperative management to prevent all maneuvers and events (e.g., external vessel compression or avulsion, hematoma, or seroma formation) that could compromise flow through the anastomosed vessel [8]. Although most complications in microsurgery are caused by technical errors and can be avoided by careful manipulation during operations [9], skill and experience are not sufficient to prevent thrombosis.

Tissue injury and vascular endothelial damage are the first steps toward thrombosis and hemostasis activation. These injuries expose tissue factor (TF) from the subendothelium and surrounding layers of the vessel. TF triggers the coagulation cascade via activation of factor VII, and this activation results in the formation of a local fibrin clot and subsequent platelet activation and aggregation [10].

The majority of compromised free flaps can be salvaged if the complication is recognized early enough and appropriate surgical interventions are made promptly. In the case of suspicious arterial or venous thrombosis, prompt intervention must be advocated to avoid irreversible tissue damage by immediately reestablishing vascularization of the pedicles $[1,2]$. For instance, Kroll et al. [1] examined the timing of free flap vascular complications and observed that about $80 \%$ of thromboses occurred within the first 2 postoperative days. A number of authors have suggested that a 2-day postoperative window is the "golden period" for potential salvage of free flaps compromised by pedicle thrombosis $[1,6,11]$. If pharmacological thrombolysis has to be performed, it would be more effective than time-consuming procedures such as thrombectomy or re-anastomosis.

Although there are abundant animal data demonstrating the benefit of thrombolytic therapy in compromised flaps $[6,7]$, pharmacological fibrinolysis has been overlooked by many reconstructive microsurgeons because of a fear of systemic complications such as hypertension or bleeding tendency. However, Lipton and Jupiter [5] first reported the use of streptokinase for successful salvage of an osteocutaneous fibular free flap that had suffered venous thrombosis. Subsequently, several studies have demonstrated a role for thrombolysis in flap salvage $[8,12]$.

Major contraindications to pharmacological fibrinolytics, to be considered only when systemic circulation of the drug is allowed, are recent stroke or malignancy (particularly if brain metastases are likely), renal insufficiency, allergy, cardiac thrombus, diabetic retinopathy, and coagulopathy [8]. If the veins of a free flap are released and the fibrinolytics are injected directly into the flap's circulation, these contraindications do not exist [10].
Furthermore, urokinase is inactivated after 15 minutes. Hence, if the venous anastomosis is performed after at least 15 minutes, various risks of systemic complications are evitable [8].

Thrombolytic agents act directly by local binding to fibrin, which activates plasmin, and activated plasmin then cleaves the fibrin within the thrombus [10]. There are three common thrombolytic drugs used in microvascular surgery-streptokinase, urokinase, and rt-PA. Group C $\beta$-hemolytic streptococcus produces streptokinase. It has been widely used in the treatment of myocardial infarction, pulmonary embolus, deep vein thrombosis, and acute limb ischemia. Streptokinase has an anaphylaxis rate of approximately $0.1 \%$ and induces antibody production. An effective dose of streptokinase in the blood is between 1,000 and 3,000 units $/ \mathrm{mL}[9,13]$. Urokinase is derived from human fetal kidney cells. It is capable of direct activation of plasminogen to plasmin, but has a short half-life of 15 minutes. The advantages of urokinase over streptokinase include: 1) less antigenicity; 2) direct plasminogen activation, allowing use in a high concentration as distinguished from streptokinase; and 3) decreased systemic effects reported clinically $[9,13]$. Rt-PA is a second generation thrombolytic agent produced by vascular endothelial cells and is a direct activator of plasminogen. Theoretically, rtPA results in less systemic bleeding complications; however, this has not been shown to be clinically significant $[9,13]$. Urokinase and rt-PA are theoretically more effective than streptokinase for dissolving an occluding thrombus.

Unfortunately, the urokinase dose adequate for safe and effective flap salvage has not been established. Anavekar et al. [14] described two cases of late vascular thrombosis in free flap, using a single bolus of 100,000 units of urokinase. In many articles different from our case, thrombolytic therapy was successfully administrated for venous and late-onset thromboses, and these applications have been mentioned above. We believe that thrombolytic therapy such as urokinase is a safe, useful, effective, and innovative tool for many cases of free flap salvage. However, microvascular surgeons must keep in mind that thrombolytic therapy neither resolves the causes for thrombosis nor eliminates the risk of re-thrombosis. Thus, eventual extrinsic or intrinsic causes for thrombosis must be removed.

The use of urokinase may provide a viable option for the treatment of suspicious intraoperative arterial thrombosis. Other fibrinolytic agents may also represent a valuable addition to the armamentarium of the microvascular surgeon. Proper technique and maximized drug delivery can help avoid systemic spread and subsequent complications. The effective dose of urokinase must be established in the near future. 


\section{REFERENCES}

1. Kroll SS, Schusterman MA, Reece GP, et al. Timing of pedicle thrombosis and flap loss after free-tissue transfer. Plast Reconstr Surg 1996;98:1230-3.

2. Khouri RK, Cooley BC, Kunselman AR, et al. A prospective study of microvascular free-flap surgery and outcome. Plast Reconstr Surg 1998;102:711-21.

3. Ozbek MR, Deune EG, Cooley BC, et al. Experimental reproduction of free flap errors: a new model of thrombosis. Ann Plast Surg 1994;32:474-7.

4. Schubert W, Hunter DW, Guzman-Stein G, et al. Use of streptokinase for the salvage of a free flap: case report and review of the use of thrombolytic therapy. Microsurgery 1987;8:117-21.

5. Lipton HA, Jupiter JB. Streptokinase salvage of a free-tissue transfer: case report and review of the literature. Plast Reconstr Surg 1987;79:977-81.

6. Romano JE, Biel MA. Thrombolysis in microvascular surgery using tissue-type plasminogen activator. Arch Otolaryngol Head Neck Surg 1989;115:1318-21.

7. Lepore DA, Knight KR, Bhattacharya S, et al. Drug mixture which improves survival of ischemic rabbit epigastric skin flaps. Microsurgery 1994;15:685-92.

8. D’Arpa S, Cordova A, Moschella F. Pharmacological thrombolysis: one more weapon for free-flap salvage. Microsurgery 2005;25:477-80.

9. Panchapakesan V, Addison P, Beausang E, et al. Role of thrombolysis in free-flap salvage. J Reconstr Microsurg 2003;19:523-30.

10. Esclamado RM, Carroll WR. The pathogenesis of vascular thrombosis and its impact in microvascular surgery. Head Neck 1999;21:355-62.

11. Betancourt FM, Mah ET, McCabe SJ. Timing of critical thrombosis after replantation surgery of the digits. J Reconstr Microsurg 1998;14:313-6.

12. Serletti JM, Moran SL, Orlando GS, et al. Urokinase protocol for free-flap salvage following prolonged venous thrombosis. Plast Reconstr Surg 1998;102:1947-53.

13. Stephan B, Schenk JF, Nemeh A, et al. The use of antithrombotic agents in microvascular surgery. Clin Hemorheol Microcirc 2009; 43:51-6.

14. Anavekar NS, Lim E, Johnston A, et al. Minimally invasive late free flap salvage: indications, efficacy and implications for reconstructive microsurgeons. J Plast Reconstr Aesthet Surg 2011;64:1517-20. 